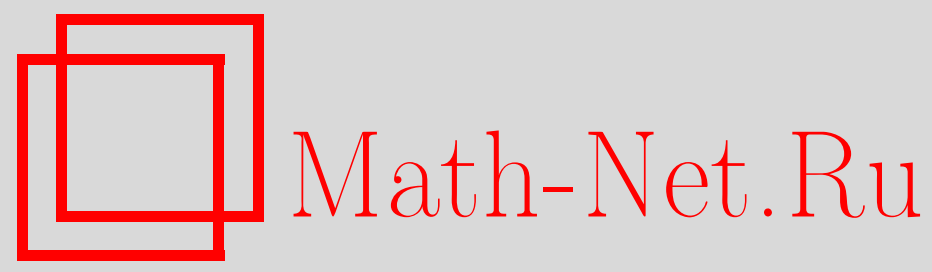

Г. А. Майлыбаева, Порядок коммуникационной сложности PIR-протоколов, Дискрет. матем., 2008, том 20, выпуск $3,136-146$

DOI: https://doi.org/10.4213/dm1020

Использование Общероссийского математического портала Math-Net.Ru подразумевает, что вы прочитали и согласны с пользовательским соглашением http://www . mathnet.ru/rus/agreement

Параметры загрузки:

IP : 54.162.85.209

26 апреля 2023 г., 14:55:06 
УДК 519.7

\title{
Порядок коммуникационной сложности PIR-протоколов
}

\author{
(c) 2008 г. Г. А. Майлыбаева
}

\begin{abstract}
Протоколы извлечения информации без раскрытия запроса (PIR-протоколы) позволяют пользователю получить желаемый бит информации из базы данных, копия которой хранится на нескольких несообщающихся серверах таким образом, что администраторы базы данных ничего не узнают о номере бита который запрашивал пользователь. Коммуникационная сложность протокола определяется как суммарное число битов, которыми обмениваются пользователь и серверы во время протокола. В работе найден порядок коммуникационной сложности PIR-протоколов в зависимости от степени существенности функций ответов серверов.
\end{abstract}

\section{1. Введение}

Рассмотрим протокол с $k+1$ участником: пользователем и $k$ несообщающимися серверами, $k \geqslant 1$, причем каждый из серверов хранит один и тот же булев вектор $x=\left(x_{0}, \ldots, x_{n-1}\right)$ длины $n$, базу данных. Пользователь желает узнать значение $i$-го бита $x_{i}$ этого вектора так, чтобы номер бита $i$ не стал известен ни одному из серверов. Протокол, который позволяет это делать, называется протоколом доступа к данным без раскрытия запроса и состоит из следующих шагов.

(1) Пользователь имеет номер бита $i$ и вырабатывает случайное число $r$. По числам $i$ и $r$ пользователь вычисляет с помощью специальной функции, называемой функцией запросов, $k$ чисел $q^{j}$ и посылает $j$-му серверу запрос $q^{j}$.

(2) Каждый из $k$ серверов по полученному запросу $q^{j}$ и базе данных $x$ с помощью специальной функции ответов вычисляет вектор $a^{j}$ и посылает его пользователю.

(3) Пользователь по числам $i, r$ и $k$ ответам серверов $a^{j}$ вычисляет с помощью реконструирующей функции нужный бит $x_{i}$.

Первое требование к протоколу состоит в том, что ни один из серверов по своему запросу $q^{j}$ не может понять, с помощью какого бита $i$ этот запрос был порожден. Это требование называется требованием защищенности. Второе требование к протоколу, называемое требованием корректности, заключается в том, что пользователь по ответам серверов правильно восстанавливает бит $x_{i}$. Предполагается, что всем участникам протокола, и пользователю и серверам, известны функции запросов, ответов и реконструирующая функция, но серверам не известно случайное число $r$ и, разумеется, не известен номер бита $i$. 
Понятие такого протокола впервые было введено в [1] под названием Private Information Retrieval, поэтому мы в дальнейшем будем называть такие протоколы PIRпротоколами.

Суммарная длина в битах запросов к серверам и ответов серверов называется коммуникационной сложностью PIR-протокола. Обозначим $C(I)$ коммуникационную сложность PIR-протокола $I$.

Известны следующие результаты. В [2] для каждого натурального $k \geqslant 2$ получен PIRпротокол с коммуникационной сложностью $n^{O(\lg \lg k /(k \lg k))}$. Для $k=2$ сложность построенного PIR-протокола равна $O\left(n^{1 / 3}\right)$. На данный момент, коммуникационная сложность этого протокола является наилучшей для $k=2$.

B [3] получен PIR-протокол для $k=3$ серверов с коммуникационной сложностью $O\left(n^{10^{-7}}\right)$. На данный момент, коммуникационная сложность этого протокола является наилучшей для 3-х серверов.

В [4] показано, что если функции ответов всех серверов линейные и длина в битах ответа любого сервера равна $a$, то длина запроса должна быть не меньше $c_{1} n 2^{-a}$, где $c$ - некоторая постоянная. Также было показано, что сложность любого PIR-протокола для 2-х серверов, функции ответов которого являются линейными и пользователь использует ровно $a$ бит из ответа каждого сервера, не превышает $c_{2} n^{1 / a+1}$, где $c_{2}$ - некоторая постоянная. Для доказательства нижних оценок авторы преобразовывают произвольный PIR-протокол в LDC-протокол (Locally Decodable Code) и приводят доказательство оценки для LDC-протокола.

В [6] показано, что если пользователь берет из ответа каждого сервера не более $a$ бит, то длина запроса должна быть не меньше $c_{3} n 2^{-6 a}$, где $c_{3}-$ некоторая постоянная. Для доказательства нижней оценки авторы преобразовывают произвольный PIR-протокол в квантовый PIR-протокол и приводят доказательство оценки для квантового PIR-протокола.

B [5] получена следующая нижняя оценка. Если функция запросов линейна и ответ любого сервера составляет ровно один бит, то длина запроса к каждому серверу должна быть не меньше $n-1$ бит. Эта оценка является точной. В той же работе построен PIRпротокол с такой же коммуникационной сложностью.

В [7] предыдущий результат был улучшен путем отказа от линейности функции запросов. А именно, было показано, что если каждый сервер посылает в ответ пользователю ровно один бит, то длина запроса должна быть не менее $n-2$.

Для того чтобы сформулировать следующий результат, дадим некоторые определения. Для любого натурального $n$ положим $E_{n}=\{0,1, \ldots, n-1\}$.

Определение 1. Пусть $k, n, s, m, a$ - произвольные натуральные числа. Пусть

$$
I=\left\langle Q, A^{0}, A^{1}, \ldots, A^{k-1}, R\right\rangle
$$

- PIR-протокол для $k$ серверов для базы данных длины $n$ с датчиком случайных чисел длины $s$ с длиной запроса $m$ и длиной ответа $a$, тогда PIR-протокол $I$ называется линейным, если для любого $j, 0 \leqslant j \leqslant k-1$, функция $A^{j}: E_{2}^{m} \rightarrow E_{2}^{a}$ линейна по каждой переменной, то есть для произвольных $b_{1}, \ldots, b_{p-1}, b, c, b_{p+1}, \ldots, b_{m} \in\{0,1\}$

$$
\begin{aligned}
& A^{j}\left(b_{1}, \ldots, b_{p-1}, b+c, b_{p+1}, \ldots, b_{m}\right)=A^{j}\left(b_{1}, \ldots, b_{p-1}, b, b_{p+1}, \ldots, b_{m}\right) \\
&+A^{j}\left(b_{1}, \ldots, b_{p-1}, c, b_{p+1}, \ldots, b_{m}\right) .
\end{aligned}
$$

Пусть $l$ - произвольное натуральное число. PIR-протокол $I$ называется $l$-мультилинейным, если для любого $j, 0 \leqslant j \leqslant k-1$, функция $A^{j}:\left(E_{2}^{l}\right)^{m / l} \rightarrow E_{2}^{a}$ линейна по 
каждой переменной, то есть для произвольных $b_{1}, \ldots, b_{p-1}, b, c, b_{p+1}, \ldots, b_{m / l}$ из $\{0,1\}^{l}$

$$
\begin{aligned}
A^{j}\left(b_{1}, \ldots, b_{p-1}, b+c, b_{p+1}, \ldots, b_{m / l}\right)=A^{j}\left(b_{1}, \ldots, b_{p-1}, b, b_{p+1}, \ldots, b_{m / l}\right) \\
\\
+A^{j}\left(b_{1}, \ldots, b_{p-1}, c, b_{p+1}, \ldots, b_{m / l}\right) .
\end{aligned}
$$

Пусть $l$ - произвольное натуральное число. PIR-протокол $I$ называется $l$-аффинным, если для любого $j, 0 \leqslant j \leqslant k-1$, функция $A^{j}:\left(E_{2}^{l}\right)^{m / l} \rightarrow E_{2}^{a}$ линейна с параметром $0^{l}=(0, \ldots, 0) \in\{0,1\}^{l}$ по каждой переменной, то есть для произвольных $b_{1}, \ldots, b_{p-1}, b, c, b_{p+1}, \ldots, b_{m / l} \in\{0,1\}^{l}$

$$
\begin{aligned}
& A^{j}\left(b_{1}, \ldots, b_{p-1}, b+c, b_{p+1}, \ldots, b_{m / l}\right)=A^{j}\left(b_{1}, \ldots, b_{p-1}, b, b_{p+1}, \ldots, b_{m / l}\right) \\
& \quad+A^{j}\left(b_{1}, \ldots, b_{p-1}, c, b_{p+1}, \ldots, b_{m / l}\right)+A^{j}\left(b_{1}, \ldots, b_{p-1}, 0^{l}, b_{p+1}, \ldots, b_{m / l}\right) .
\end{aligned}
$$

B [8] показано, что любой линейный PIR-протокол для $k$ серверов имеет сложность не менее $c_{4} \sqrt{n /(2 k)}$, где $c_{4}-$ некоторая постоянная. Пусть $k, l-$ произвольные натуральные числа и $\varepsilon-$ произвольное положительное число. Тогда если $I$ произвольный $l$-мультилинейный или $l$-аффинный PIR-протокол для $k$ серверов, то почти для всех $n$

$$
C(I) \geqslant\left(1 /(k-1)^{1 /(l+1)}-1\right) n^{1 /(l+1)} .
$$

В [9] получена нижняя оценка для билинейных, основанных на группах (group based) PIR-протоколов с двумя серверами. Реконструирующая функция билинейных PIR-протоколов является суммой бит ответов серверов, а то, что протоколы основаны на группах означает, что серверы представляют базу данных в виде функции, действующей в конечной группе, и позволяют пользователю запросить значение этой функции на некотором элементе группы, используя схему разделения секрета в этой группе. А именно, показано, что для данного класса PIR-протоколов

$$
C(I) \geqslant \Omega\left(n^{1 / 3}\right) .
$$

В данной работе получен результат для более широкого класса PIR-протоколов. A именно, во-первых, в отличие от всех рассмотренных выше результатов, мы не предполагаем, что длины ответов серверов должны быть равны между собой, во-вторых, мы не предполагаем, что пользователь использует из ответа каждого из серверов одинаковое число битов. В-третьих, и это самое главное отличие, нижняя оценка, полученная в данной работе, не требует линейности ни функции запросов, ни функции ответов, а также не накладывает никаких ограничений на реконструирующую функцию. Также заметим, что для доказательства нижней оценки, авторы статьи [4] используют сведение PIR-протоколов к LDC-протоколам, а авторы статьи [6] используют сведение PIR-протоколов к квантовым PIR-протоколам. В данной статье мы доказываем нижнюю оценку напрямую для PIR-протоколов.

Определим степень существенности функции ответов сервера как максимальное число битов базы данных, которые может использовать сервер для получения одного бита ответа. В работе найден порядок коммуникационной сложности PIR-протоколов в зависимости от степени существенности функций ответов серверов.

Автор благодарен Э. Э. Гасанову за постановку задачи. 


\section{2. Основные понятия}

Формальное определение PIR-протокола было введено в [10, 11].

Для натурального $n$ введем обозначение

$$
E_{n}=\{0,1, \ldots, n-1\} .
$$

Пусть $k, n, s, p^{0}, \ldots, p^{k-1}-$ натуральные числа, $p=p^{0}+\ldots+p^{k-1}$. Пусть на множестве

$$
B=\left\{(i, r), i \in E_{n}, r \in E_{s}\right\}
$$

задано вероятностное пространство $\left\langle B, 2^{B}, \mathbf{P}\right\rangle$, где $\mathbf{P}(i, r)=1 /(n s)$ для любых $i \in E_{n}$, $r \in E_{s}$. Тогда $(k, n, s, p)$ PIR-протоколом называется набор из $k+2$ функций $I=\left\langle Q, A^{0}, \ldots, A^{k-1}, R\right\rangle$, где $Q, A^{0}, \ldots, A^{k-1}, R-$ некоторые отображения, $Q: E_{k} \times E_{n} \times E_{s} \rightarrow E_{s}, A^{j}: E_{s} \times\{0,1\}^{n} \rightarrow\{0,1\}^{p^{j}}, j \in E_{k}, R: E_{n} \times E_{s} \times\{0,1\}^{p} \rightarrow\{0,1\}$ такие, что выполнены следующие два условия.

Условие корректности: для любых $i \in E_{n}, r \in E_{s}$

$$
R\left(i, r, A^{0}(Q(0, i, r), x), \ldots, A^{k-1}(Q(k-1, i, r), x)\right)=x_{i} .
$$

Условие защищенности: для любых $q \in E_{s}, t \in E_{k}, i, j \in E_{n}$

$$
\mathbf{P}(Q(t, i, r)=q)=\mathbf{P}(Q(t, j, r)=q) .
$$

Здесь и везде далее в статье $k$ - число серверов, $n$ - длина базы данных $x=\left(x_{0}, x_{1}, \ldots, x_{n-1}\right), s-$ параметр датчика случайных чисел, точнее, датчик случайных чисел дает равновероятно числа из множества $E_{s}, p^{j}-$ число битов в ответе $j$-го сервера, $A^{j}$ - функция ответа $j$-го сервера, $j \in E_{k}, R-$ реконструирующая функция.

Содержательно протокол $I=\left\langle Q, A^{0}, \ldots, A^{k-1}, R\right\rangle$ состоит из следующих шагов.

Пользователь $U$, имея запрос $i$, вырабатывает случайное число $r \in E_{s}$, для каждого $j \in E_{k}$ вычисляет $q^{j}=Q(j, i, r)$ и посылает $q^{j}$ серверу $S_{j}$.

Каждый сервер $S_{j}, j \in E_{k}$, вычисляет $a^{j}=\left(a_{0}^{j}, \ldots, a_{p^{j}-1}^{j}\right)=A^{j}\left(q^{j}, x\right)$ и посылает вектор $a^{j}$ пользователю.

Пользователь $U$ вычисляет $x_{i}=R\left(i, r, a^{0}, \ldots, a^{k-1}\right)$.

Если $d-$ вещественное число, то через ] $d$ [ обозначим наименьшее целое не меньшее, чем $d$, а через $[d]$ - наибольшее целое не большее, чем $d$.

Величина

$$
C(I)=k] \log _{2} s[+p
$$

называется коммуникационной сложностью протокола $I$ и представляет собой число битов, переданных в процессе протокола.

Условие корректности гарантирует, что пользователь получит нужный бит базы данных, а условие защищенности, что ни один из серверов по числу $q$, которое он получил, не сможет понять, какой бит интересует пользователя.

Основной целью исследований в этой области является построение для заданного числа серверов $k$, заданной длины базы данных $n$ и максимального значения датчика случайных чисел $s$ PIR-протокола с минимальной коммуникационной сложностью.

Степенью существенности булевой функции $f\left(x_{0}, \ldots, x_{l-1}\right)$ назовем число переменных, от которых она существенно зависит, и обозначим его через $S(f)$. 
Степенью существенности булевой вектор-функции

$$
F\left(x_{0}, \ldots, x_{l-1}\right)=\left(f_{0}\left(x_{0}, \ldots, x_{l-1}\right), \ldots, f_{m-1}\left(x_{0}, \ldots, x_{l-1}\right)\right)
$$

назовем число

$$
S(F)=\max _{0 \leqslant t \leqslant m-1} S\left(f_{t}\right) .
$$

Пусть $A^{j}(q, x)=\left(A_{0}^{j}(q, x), \ldots, A_{p^{j}-1}^{j}(q, x)\right)$. Для функций $A^{j}(q, x), A_{l}^{j}(q, x), l \in E_{p^{j}}$, $j \in E_{k}, q \in E_{s}$, также будем использовать следующую запись:

$$
A^{j}(q, x)=A^{j}(q)(x), \quad A_{l}^{j}(q, x)=A_{l}^{j}(q)(x),
$$

где $A^{j}(q):\{0,1\}^{n} \rightarrow\{0,1\}^{p^{j}}$ - булева вектор-функция, а $A_{l}^{j}(q):\{0,1\}^{n} \rightarrow\{0,1\}-$ булева функция $n$ переменных.

Степенью существенности функции ответов $j$-го сервера

$$
A^{j}: E_{s} \times\{0,1\}^{n} \rightarrow\{0,1\}^{p^{j}}, \quad j \in E_{k},
$$

назовем число

$$
S\left(A^{j}\right)=\max _{q \in E_{S}} S\left(A^{j}(q)\right) .
$$

Обозначим $\mathscr{I}(k, n, s)$ класс всех $(k, n, s, p)$ PIR-протоколов, где $p>0$. Пусть $\mathscr{A}-$ некоторое множество PIR-протоколов. Положим также

$$
\begin{aligned}
C(k, n, s, \mathscr{A}) & =\min \{C(I): I \in \mathscr{A} \cap \mathscr{I}(k, n, s)\}, \\
C(k, n, \mathscr{A}) & =\min _{s \in \mathbf{N}} C(k, n, s, \mathscr{A}) .
\end{aligned}
$$

Для натурального $d$ обозначим через $\mathscr{A}_{d}$ множество всех PIR-протоколов таких, что степень существенности функции ответов каждого сервера не превосходит $d$.

Теорема 1. Для любых натуральных $k, n$ u $d$ mаких, что $d \leqslant n^{2 k-2 / 2 k-1}$,

$$
C\left(k, n, 2^{(k-1)\left[d^{1 / 2 k-2}\right]}, \mathscr{A}_{d}\right) \leqslant\left(k^{2}-k\right) d^{1 / 2 k-2}+4 k n / d .
$$

Теорема 2. Для любых натуральных $k, n, s, d$

$$
\left.C\left(k, n, s, \mathcal{A}_{d}\right) \geqslant k\right] \log _{2} s[+n / d .
$$

Следствие 1. Если натуральные числа $k, n, d$ таковы, что $d \preccurlyeq n^{2 k-2 / 2 k-1} n$ ри $n \rightarrow \infty$, то при $n \rightarrow \infty$

$$
C\left(k, n, \mathscr{A}_{d}\right) \asymp n / d .
$$

\section{3. Верхняя оценка}

Рассмотрим класс PIR-протоколов $I^{p o l}$ для $k \geqslant 2$ серверов, построенных по алгоритму, описанному в [2]. Используя общую балансирующую технику, описанную в [1], для любых натуральных $n$ и $k$ построим PIR-протокол с любой, наперед заданной степенью существенности функции ответов, не превосходящей $n^{2 k-2 / 2 k-1}$.

Пусть $m-$ минимальное натуральное число такое, что $\left(\begin{array}{c}m \\ 2 k-1\end{array}\right) \geqslant n$. Для произвольного вектора $y=\left(y_{0}, \ldots, y_{m-1}\right) \in E_{2}^{m}$ обозначим $H\left(y_{0}, \ldots, y_{m-1}\right)=\left|\left\{l \in E_{m}: y_{l}=1\right\}\right|$ число единиц в векторе $y$. Для любого $i \in E_{n}$ положим $E(i)=\left(E_{0}(i), \ldots, E_{m-1}(i)\right) \in E_{2}^{m}$, причем $H(E(i))=2 k-1$ и $E\left(i_{1}\right) \neq E\left(i_{2}\right)$ для любых $i_{1}, i_{2} \in E_{n}$ таких, что $i_{1} \neq i_{2}$. По выбору $m$ ясно, что всегда найдется $n$ векторов, удовлетворяющих этим условиям.

Протокол проходит следующим образом. 
Шаг 1. Пользователь хочет узнать значение $x_{i}, i \in E_{n}$, выбирает случайные векторы $y_{j}=\left(y_{j, 0}, \ldots, y_{j, m-1}\right) \in E_{2}^{m}, j \in E_{k}$ такие, что

$$
\sum_{j \in E_{k}} y_{j}=\left(\sum_{j \in E_{k}} y_{j, 0}, \ldots, \sum_{j \in E_{k}} y_{j, m-1}\right)=\left(E_{0}(i), \ldots, E_{m-1}(i)\right)=E(i),
$$

и посылает серверу $S_{j}$ векторы $y_{l}, l \in E_{k}\{j\}$.

Шаг 2. Сервер $S_{j}, j \in E_{k}$ по базе данных $x=\left(x_{0}, \ldots, x_{n-1}\right)$ строит многочлен, коэффициентами которого являются биты базы данных

$$
P_{x}\left(Y_{0}, \ldots, Y_{m-1}\right)=\sum_{i \in E_{n}} x_{i} p_{i}\left(Y_{0}, \ldots, Y_{m-1}\right)
$$

где $Y_{0}, \ldots, Y_{m-1} \in E_{2}$. Многочлены $p_{0}, \ldots, p_{n-1}$ определяются рекурсивно следующим образом.

Для каждого $i \in E_{n}$ пусть $B_{i}=\left\{l: E_{l}(i)=1\right\}$ - множество позиций в векторе $E(i)$, в которых стоят единицы. Тогда для любого $i \in E_{n}$

$$
p_{i}\left(Y_{0}, \ldots, Y_{m-1}\right)=\prod_{h \in B_{i}} Y_{h}
$$

Ясно, что для любых $i, g \in E_{n}$

$$
p_{i}(E(g))= \begin{cases}1, & \text { если } g=i, \\ 0, & \text { если } g \neq i .\end{cases}
$$

Тогда для любого $i \in E_{n}$

$$
P_{x}(E(i))=\sum_{g \in E_{n}} x_{g} p_{g}(E(i))=x_{i} .
$$

Определим многочлен $q(\tilde{Y})$ от $k m$ переменных степени $2 k-1$, полагая

$$
q(\tilde{Y})=q\left(\left\{Y_{j, b}\right\}_{j \in E_{k}, b \in E_{m}}\right)=P_{x}\left(\sum_{j \in E_{k}} Y_{j, 0}, \ldots, \sum_{j \in E_{k}} Y_{j, m-1}\right) .
$$

Для произвольного $i \in E_{n}$ рассмотрим многочлен

$$
q_{i}(\tilde{Y})=q_{i}\left(\left\{Y_{j, b}\right\}_{j \in E_{k}, b \in E_{m}}\right)=p_{i}\left(\sum_{j \in E_{k}} Y_{j, 0}, \ldots, \sum_{j \in E_{k}} Y_{j, m-1}\right)
$$

от $k m$ переменных степени $2 k-1$. Ясно, что

$$
q(\tilde{Y})=\sum_{i \in E_{n}} x_{i} q_{i}(\tilde{Y})
$$

Пусть $B_{i}=\left\{b_{1}^{i}, \ldots, b_{2 k-1}^{i}\right\}$, тогда любой одночлен $q_{i}$ имеет вид $Y_{j_{1}, b_{1}^{i}} \ldots Y_{j_{2 k-1}, b_{2 k-1}^{i}}$, где $j_{1}, \ldots, j_{2 k-1} \in E_{k}$. 
Пусть $Y_{j_{1}, b_{1}^{i}} \ldots Y_{j_{2 k-1}, b_{2 k-1}^{i}}$ - произвольный одночлен многочлена $q_{i}$. Рассмотрим набор $\left\{j_{1}, \ldots, j_{2 k-1}\right\}$. Поскольку в наборе ровно $2 k-1$ элементов, каждый из которых принимает значение $j_{1}, \ldots, j_{2 k-1} \in E_{k}$, существует число $j \in E_{k}$, которое встречается в этом наборе не более чем $[2 k-1 / k]=1$ раз. Учитывая, что серверу $S_{j}$ известны значения всех переменных вида $Y_{l, b}, l \in E_{k}\{j\}, b \in E_{m}$ и неизвестны значения переменных $Y_{j, 0}, \ldots, Y_{j, m-1}$, получаем, что сервер $S_{j}$ не знает значение не более 1 переменной из этого одночлена. Поставим этот одночлен в соответствие серверу $S_{j}$. Проделаем эту операцию с каждым одночленом $q_{i}$

Обозначим сумму всех одночленов $q_{i}$, поставленных в соответствие серверу $S_{j}$ через $q_{i}^{j}$. Тогда многочлен, соответствующий серверу $S_{j}$, равен $q^{j}=\sum_{i \in E_{n}} x_{i} q_{i}^{j}$. Заметим, что $q=\sum_{j \in E_{k}} q^{j}$ и каждый одночлен $q$ принадлежит ровно одному из многочленов $q^{j}$.

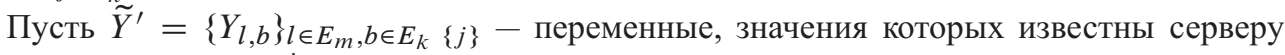
$S_{j}$, тогда многочлен $q_{i}^{j}$, соответствующий серверу $S^{j}$, имеет вид

$$
q_{i}^{j}(\tilde{Y})=q_{i}^{\prime j}(\tilde{Y})+q_{i}^{\prime \prime}\left(\tilde{Y}^{\prime}\right),
$$

где $j \in\left\{j_{1}, \ldots, j_{2 k-1}\right\}$ для любого одночлена $Y_{j_{1}, b_{1}^{i}} \ldots Y_{j_{2 k-1}, b_{2 k-1}^{i}}$ многочлена $q_{i}^{\prime j}(\tilde{Y})$, и многочлен $q_{i}^{\prime \prime}{ }_{i}^{j}\left(\tilde{Y}^{\prime}\right)$ зависит от переменных $\tilde{Y}^{\prime}$, значения которых известны серверу $S_{j}$.

Сервер $S_{j}$, подставив значения переменных, которые ему известны, в многочлен $q^{j}$, получает линейный многочлен $\hat{q}^{j}$ от переменных $Y_{j, b}, b \in E_{m}$ вида

$$
\hat{q}^{j}=t_{0} Y_{j, 0}+\ldots+t_{m-1} Y_{j, m-1}+t_{m},
$$

где $t_{l}=t_{l}\left(x, \tilde{Y}^{\prime}\right), l \in E_{m+1}$, причем $t_{m}$ имеет вид

$$
t_{m}=\sum_{i \in E_{n}} x_{i} q_{i}^{\prime \prime}\left(\tilde{Y}^{\prime}\right) .
$$

До настоящего момента мы в точности повторили протокол, описанный в [2], для случая $H(E(i))=2 k-1$, где $i \in E_{n}$. Для уменьшения степени существенности функции ответов серверов, произведем небольшую модификацию протокола.

Пусть $[n / m]=n^{\prime}$. Представим $t_{m}$ в виде суммы $t_{m}=t_{0}^{\prime}+\ldots+t_{m}^{\prime}$, где для любого $l$, $0 \leqslant l \leqslant m-1$,

$$
t_{l}^{\prime}=\sum_{l n^{\prime} \leqslant i<(l+1) n^{\prime}} x_{i} q_{i}^{\prime \prime}{ }_{i}^{j}\left(\tilde{Y}^{\prime}\right), \quad t_{m}^{\prime}=\sum_{m n^{\prime} \leqslant i<n} x_{i} q_{i}^{\prime \prime}{ }_{i}^{j}\left(\tilde{Y}^{\prime}\right) .
$$

Определим функцию ответов $A^{j}(q, x)$ сервера $S_{j}, j \in E_{k}$, полагая

$$
A^{j}(q, x)=\left(t_{0}\left(x, \tilde{Y}^{\prime}\right), \ldots, t_{m-1}\left(x, \tilde{Y}^{\prime}\right), t_{0}^{\prime}\left(x, \tilde{Y}^{\prime}\right), \ldots, t_{m}^{\prime}\left(x, \tilde{Y}^{\prime}\right)\right) .
$$

Шаг 3. Пользователю известны значения всех переменных $Y_{j, 0}, \ldots, Y_{j, m-1}, j \in E_{k}$, он может вычислить

$$
\sum_{j \in E_{k}} q^{j}\left(y_{j, 0}, \ldots, y_{j, m-1}\right)=P_{x}\left(\sum_{j \in E_{k}} y_{j, 0}, \ldots, \sum_{j \in E_{k}} y_{j, m-1}\right)=P_{x}(E(i))=x_{i} .
$$

Коммуникационная сложность протокола

$$
C\left(I^{p o l}\right) \leqslant k(k-1) m+k(2 m+1)=k^{2} m+2 k m+k .
$$


Положим $m=\left[n^{1 / 2 k-1}\right]$, такого числа переменных достаточно для выполнения неравенства $\left(\begin{array}{c}m \\ 2 k-1\end{array}\right) \geqslant n$. Тогда

$$
C\left(I^{p o l}\right) \leqslant k^{2} m+2 k m+k \leqslant k^{2} n^{1 / 2 k-1}+2 k n^{1 / 2 k-1}+k=O\left(n^{1 / 2 k-1}\right) .
$$

Чтобы на первом шаге получить $k$ случайных векторов $y_{j}=\left(y_{j, 0}, \ldots, y_{j, m-1}\right), j \in E_{k}$, принадлежащих $E_{2}^{m}$ и в сумме дающих вектор $E(i)$, пользователь вырабатывает $k-1$ случайный вектор, $k$-й вектор получается путем сложения всех случайных векторов и вектора $E(i)$. Таким образом, длина датчика случайных чисел равна $s=2^{(k-1) m} \leqslant 2^{(k-1) n^{1 / 2 k-1}}$.

Найдем степень существенности функций ответов $A^{j}, j \in E_{k}$. Ясно, что для любого $0 \leqslant l \leqslant m$ степень существенности функции $t_{l}^{\prime}\left(\tilde{Y}^{\prime}\right)(x)=t_{l}^{\prime}\left(\tilde{Y}^{\prime}, x\right)$ не превосходит $S\left(t_{l}^{\prime}\left(\tilde{Y}^{\prime}\right)\right) \leqslant n / m$.

Рассмотрим функции $t_{l}, l \in E_{m}$. Для всех $j \in E_{k}, b \in E_{m}$ подсчитаем число многочленов $q_{i}$, в которых может присутствовать одночлен вида $Y_{j, b} Y_{j_{1}, b_{1}} \ldots Y_{j_{2 k-2}, b_{2 k-2}}$, где $j_{1}, \ldots, j_{2 k-1} \in E_{k}, b_{1}, \ldots, b_{2 k-1} \in E_{m}$. Для того, чтобы многочлен $q_{i}$ содержал одночлен такого вида, необходимо и достаточно, чтобы многочлен $p_{i}$ содержал одночлен вида $Y_{b} Y_{b_{1}} \ldots Y_{b_{2 k-2}}$, где $b_{1}, \ldots, b_{2 k-1} \in E_{m}$ - произвольные числа. По определению многочлена $p_{i}=\prod_{h \in B_{i}} Y_{h}$ для этого необходимо и достаточно, чтобы $b \in B_{i}$, то есть чтобы $E_{b}(i)=1$. Учитывая, что $H(E(i))=2 k-1$ для любого $i \in E_{n}$, получаем, что число векторов $E(i)$, удовлетворяющих этому условию, равно

$$
\left(\begin{array}{c}
m-1 \\
2 k-2
\end{array}\right)=\frac{1}{m}\left(\begin{array}{c}
m \\
2 k-1
\end{array}\right) \leqslant \frac{n}{m} .
$$

Таким образом, для любого $j \in E_{k}$ степень существенности функции ответов $A^{j}$ не превосходит

$$
S\left(A^{j}\right)=\max _{t \in E_{m}} A_{t}^{j} \leqslant \frac{n}{m} \leqslant n^{2 k-2 /(2 k-1)} .
$$

Доказательство теоремы 1. Для натуральных $k, n, d$ таких, что $d \leqslant n^{2 k-2 / 2 k-1}$, опишем PIR-протокол $I_{d}^{p o l}=\left\langle Q^{\prime}, A^{\prime 0}, \ldots, A^{\prime k-1}, R^{\prime}\right\rangle$ с датчиком случайных чисел, принимающим $s=2^{(k-1)\left[d^{1 / 2 k-2}\right]}$ значений, степень существенности функций ответов серверов которого не превосходит $d$.

Протокол состоит в следующем. Без ограничения общности будем считать, что $n$ делится нацело на $\left[d^{2 k-1 / 2 k-2}\right]$, в противном случае дополним базу данных до нужной длины нулями. База данных $x=\left(x_{0}, \ldots, x_{n-1}\right)$ делится на $l=n /\left[d^{2 k-1 / 2 k-2}\right]$ частей, длина каждой из которых равна $n^{\prime}=\left[d^{2 k-1 / 2 k-2}\right]$, а именно, $E_{n}=\bigcup_{t \in E_{l}} I_{t}$, где $I_{t}=\left\{t\left[d^{2 k-1 / 2 k-2}\right], \ldots,(t+1)\left[d^{2 k-1 / 2 k-2}\right]-1\right\}$ для любого $t \in E_{l}$

К каждой из $l$ частей базы данных применяем протокол $I^{p o l}$ для базы данных длины $n^{\prime}$. Тогда степень существенности функции ответов протокола в каждой части будет не превышать $n^{\prime 2 k-2 / 2 k-1} \leqslant\left(\left[d^{2 k-1 / 2 k-2}\right]\right)^{2 k-2 / 2 k-1} \leqslant d$.

Положим $m^{\prime}=\left[d^{1 / 2 k-2}\right]$, тогда будет выполнено условие $\left(\begin{array}{c}m^{\prime} \\ 2 k-1\end{array}\right) \geqslant n^{\prime}$. Для любого $i \in E_{n^{\prime}}$ определим вектор $E(i) \in E_{2}^{m^{\prime}}$, такой, что $H(E(i))=2 k-1$ и $E\left(i_{1}\right) \neq E\left(i_{2}\right)$ для любых $i_{1}, i_{2} \in E_{n^{\prime}}, i_{1} \neq i_{2}$. По выбору $m^{\prime}$ ясно, что всегда найдется $n^{\prime}$ векторов, удовлетворяющих этим условиям.

Шаг 1. Пользователь хочет узнать значение $x_{i}, i \in E_{n}$, он строит запрос, используя функцию запросов протокола $I^{\text {pol }}$ для базы данных длины $n^{\prime}$ и номера 
искомого бита $i^{\prime}=i \bmod n^{\prime}$. Затем пользователь выбирает случайные векторы $y_{j}=\left(y_{j, 0}, \ldots, y_{j, m^{\prime}-1}\right) \in E_{2}^{m^{\prime}}, j \in E_{k}$, такие, что

$$
\sum_{j \in E_{k}} y_{j}=\left(\sum_{j \in E_{k}} y_{j, 0}, \ldots, \sum_{j \in E_{k}} y_{j, m^{\prime}-1}\right)=\left(E_{0}\left(i^{\prime}\right), \ldots, E_{m^{\prime}-1}\left(i^{\prime}\right)\right)=E\left(i^{\prime}\right),
$$

и посылает серверу $S_{j}$ векторы $y_{l}, l \in E_{k} \backslash\{j\}$.

Шаг 2. Для каждой части $I_{t}, t \in E_{l}$, сервер $S_{j}, j \in E_{k}$, вычисляет ответ согласно протоколу $I^{p o l}$. Ответом сервера $S_{j}$ является вектор $a^{j}=\left(a_{0}^{j}, \ldots, a_{p^{j}-1}^{j}\right)$, где $p^{j}=\left(2 m^{\prime}+1\right) n / n^{\prime}$.

Шаг 3. Для того, чтобы вычислить бит $x_{i}$, пользователь выбирает $t \in E_{l}$ такое, что $i \in I_{t}$, и вычисляет ответ согласно протоколу $I^{p o l}$, используя ответы серверов $S_{j}$, $j \in E_{k}$, вычисленных для базы данных с индексами из $I_{t}$.

Коммуникационная сложность протокола равна

$$
\begin{aligned}
C\left(I_{d}^{p o l}\right) & =k(k-1) m^{\prime}+k\left(2 m^{\prime}+1\right) n / n^{\prime} \leqslant k(k-1) m^{\prime}+2 k m^{\prime} n / n^{\prime} \\
& \leqslant k(k-1) d^{1 / 2 k-2}+2 k d^{1 / 2 k-2} \frac{n}{d^{2 k-1 / 2 k-2}+1} \\
& \leqslant\left(k^{2}-k\right) d^{1 / 2 k-2}+4 k n / d .
\end{aligned}
$$

\section{4. Нижняя оценка}

Доказательство теоремы 2. Рассмотрим произвольный $(k, n, s, p)$ PIR-протокол

$$
I=\left\langle Q, A^{0}, \ldots, A^{k-1}, R\right\rangle \in \mathscr{A}_{d} .
$$

Пусть

$$
p=\sum_{j \in E_{k}} p^{j}
$$

Для произвольной булевой функции $n$ переменных $f\left(x_{0}, \ldots, x_{l-1}\right)$ обозначим $V(f) \subseteq E_{l}$ множество ее существенных переменных, тогда $S(F)=|V(f)|-$ степень существенности функции $f$. Для произвольной булевой вектор-функции $n$ переменных $F\left(f_{0}\left(x_{0}, \ldots, x_{l-1}\right), \ldots, f_{m-1}\left(x_{0}, \ldots, x_{l-1}\right)\right)$ пусть

$$
V(F)=\bigcup_{t \in E_{m}} V\left(f_{t}\right) \subseteq E_{l},
$$

тогда $W(F)=|V(F)|$ - совокупная степень существенности функции $F$.

Рассмотрим булеву вектор-функцию

$$
A(i, r, x)=\left(A^{0}(Q(0, i, r), x), \ldots, A^{k-1}(Q(k-1, i, r), x)\right) .
$$

Учитывая, что для любых $j \in E_{k}, l \in E_{p^{j}}$ функции $A_{l}^{j}$ являются существенными функциями не более чем $d$ переменных, $\left(A_{l}^{j}\right) \leqslant d$, получаем, что

$$
W(A) \leqslant \sum_{j \in E_{k}} W\left(A^{j}\right) \leqslant \sum_{j \in E_{k}} \sum_{l \in E_{p^{j}}} S\left(A_{l}^{j}\right) \leqslant \sum_{j \in E_{k}} p^{j} d=p d .
$$


Пусть отображение $R^{\prime}: E_{n} \times E_{s} \times\{0,1\}^{p} \rightarrow\{0,1\}$ задано равенствами

$$
R^{\prime}(i, r, A(i, r, x))=R\left(i, r, A^{0}(Q(0, i, r), x), \ldots, A^{k-1}(Q(k-1, i, r), x)\right)=x_{i} .
$$

Предположим, что $p d<n$, тогда $W(A)<n$, и значит, существует число $i \in E_{n}$ такое, что $x_{i}$ не является существенной переменной $A(i, r, x)$. А именно, для любых соседних по $i$-й компоненте векторов $x^{\prime}, x^{\prime \prime} \in\{0,1\}^{n}$ (векторов, для которых $x_{l}^{\prime}=x_{l}^{\prime \prime}$ для любого $l \in E_{n} \backslash\{i\}$ и $x_{i}^{\prime} \neq x_{i}^{\prime \prime}$ ) для любого $r \in E_{s}$ справедливо равенство

$$
A\left(i, r, x^{\prime}\right)=A\left(i, r, x^{\prime \prime}\right) .
$$

Тогда

$$
x_{i}^{\prime}=R^{\prime}\left(i, r, A\left(i, r, x^{\prime}\right)\right)=R^{\prime}\left(i, r, A\left(i, r, x^{\prime \prime}\right)\right)=x_{i}^{\prime \prime} .
$$

Это противоречие доказывает, что $p d \geqslant n$. Тогда

$$
C(I)=k] \log _{2} s[+p \geqslant k] \log _{2} s[+n / d .
$$

\section{5. Порядок коммуникационной сложности в классе $\mathscr{A}_{d}$}

Рассмотрим произвольные натуральные $k, d, n$ и $s$ такие, что $d \preccurlyeq n^{2 k-2 / 2 k-1}$ при $n \rightarrow \infty$ и $s=2^{(k-1)\left[d^{1 / 2 k-2}\right]}$. Тогда из теоремы 1 следует неравенство

$$
C\left(k, n, \mathscr{A}_{d}\right) \leqslant C\left(k, n, s, \mathscr{A}_{d}\right) \leqslant\left(k^{2}-k\right) d^{1 / 2 k-2}+4 k \frac{n}{d} \preccurlyeq \frac{n}{d} .
$$

Из теоремы 2 следует неравенство

$$
\left.C\left(k, n, \mathscr{A}_{d}\right) \geqslant C\left(k, n, s, \mathscr{A}_{d}\right) \geqslant k\right] \log _{2} s\left[+\frac{n}{d} \geqslant \frac{n}{d} .\right.
$$

Следовательно, при $n \rightarrow \infty$ и $d \preccurlyeq n^{2 k-2 / 2 k-1}$

$$
C\left(k, n, \mathscr{A}_{d}\right) \asymp n / d .
$$

\section{Список литературы}

1. Chor B., Goldreich O., Kushilevitz E., Sudan M., Private information retrieval. In: Proc. 36th IEEE Symposium on Foundations of Computer Science. IEEE Computer Society Press, 1995, pp. 41-51.

2. Beimel A., Ishai Y., Kushilevitz E., Raymond J.-F., Breaking the $O\left(n^{1 /(2 k-1)}\right)$ barrier for information-theoretic private information retrieval. In: Proc. 43rd IEEE Symposium on Foundations of Computer Science. IEEE Computer Society Press, 2002, pp. 261-270.

3. Yekhanin S., New locally decodable codes and private information retrieval schemes. Electronic Colloquium on Computational Complexity (TR06-127).

4. Goldreich O., Karloff H., Schulman L., Trevisan L., Lower bounds for linear locally decodable codes and private information retrieval systems. In: Proc. 17th IEEE Conference on Computational Complexity. IEEE Computer Society Press, 2002, pp. 175-183.

5. Chor B., Goldreich O., Kushilevitz E., Sudan M., Private information retrieval. J. ACM (1998) 45, 965-981.

6. Kerendis I., de Wolf R., Exponential lower bound for 2-query locally decodable codes. In: Proc. 35th ACM Symposium on Theory of Computing. ACM, 2003, pp. 106-115. 
7. Beigel R., Fortnow L., Gasarch W., A nearly tight lower bound for private information retrieval protocols. Electronic Colloquium on Computational Complexity (TR03-087).

8. Itoh T., On lower bounds for the communication complexity of private information retrieval. IEICE Trans. Fundamentals of Electronics, Commun. and Comp. Sci. (2001) ES4-A, №1, 157-164.

9. Razborov A., Yekhanin S., An $\Omega\left(n^{1 / 3}\right)$ lower bound for bilinear group based private information retrieval. Electronic Colloquium on Computational Complexity (TR06-050).

10. Гасанов Э. Э., Майлыбаева Г. А., Доступ к базам данных без раскрытия запроса. В сб.: Материаль конференции «Математика и безопасные информационные технологии», Москва, 2003, c. 393-395.

11. Майлыбаева Г. А., Границы вырожденности протоколов доступа к данным без раскрытия запроса. Дискретная математика (2006) 18, №2, 98-110.

Статья поступила 3.08.2006. 